\title{
Amplitudes at Weak Coupling as Polytopes in $\operatorname{AdS}_{5}$
}

\author{
Lionel Mason* \& David Skinner ${ }^{\dagger}$ \\ * The Mathematical Institute, \\ 24-29 St. Giles', Oxford, OX1 3LB, \\ United Kingdom \\ ${ }^{\dagger}$ Perimeter Institute for Theoretical Physics, \\ 31 Caroline St., Waterloo, ON, N2L $2 Y 5$, \\ Canada
}

\begin{abstract}
We show that one-loop scalar box functions can be interpreted as volumes of geodesic tetrahedra embedded in a copy of $\mathrm{AdS}_{5}$ that has dual conformal space-time as boundary. When the tetrahedron is space-like, it lies in a totally geodesic hyperbolic three-space inside $\mathrm{AdS}_{5}$, with its four vertices on the boundary. It is a classical result that the volume of such a tetrahedron is given by the Bloch-Wigner dilogarithm we show that this agrees with the standard physics formulæ for such box functions. The combinations of box functions that arise in the $n$-particle one-loop MHV amplitude in $\mathcal{N}=4$ super YangMills correspond to the volume of a three-dimensional polytope without boundary, all of whose vertices are attached to a null polygon (which in other formulations is interpreted as a Wilson loop) at infinity.
\end{abstract}




\section{Introduction}

A key property of planar scattering amplitudes in $\mathcal{N}=4$ super Yang-Mills is the existence of a 'dual' copy of the conformal group. While the usual conformal group acts on the compactification of space-time, this dual conformal group acts on the compactified space of region momenta.

The dual conformal group first emerged in studies [1-3] of various integrals that contribute to planar amplitudes, and was used in [4] to construct an ansatz for the fourparticle amplitude at five loops. Its significance was greatly enhanced by the work of Alday \& Maldacena [5], calculating the strong coupling limit of planar amplitudes from the area of a minimal surface (string worldsheet) embedded in a copy of five dimensional anti-de Sitter space whose boundary is the compactified space of region momenta. Dual conformal symmetry was then studied systematically at weak coupling in [6-11, and its presence understood as a reflection of the integrability of planar $\mathcal{N}=4$ super Yang-Mills in the amplitude sector [12 17]. Infra-red divergences render this symmetry anomalous, but it is conjectured to be an exact symmetry of the loop integrand.

In [18], Hodges introduced twistors for the dual conformal groupl, naming them momentum twistors since they relate to the conformal geometry of momentum space. Hodges showed that NMHV tree amplitudes could be represented as volumes of polytopes in momentum twistor space, providing a geometric understanding of the cancellation of spurious singularities present in individual BCFW terms. Subsequently, a general representation in momentum twistor space was found, conjecturally for all tree amplitudes and leading singularities in [19] using a contour integral over an auxiliary Grassmannian. This Grassmannian integral is analogous and equivalent to the Grassmannian representation of tree amplitudes and leading singularities based on ordinary twistors for the space-time superconformal group [20,21].

At one-loop, Passarino-Veltman reduction can be used to decompose four-dimensional scattering amplitudes into momentum space scalar integrals usually represented as boxes, triangles and bubbles. For $\mathcal{N}=4$ super Yang-Mills only boxes appear [22]. Very recently, these box functions have been studied using momentum twistors [23]. The purpose of this note is to show how this analysis leads to a new geometric interpretation of box functions as volumes of three-dimensional tetrahedra in the five dimensional anti-de Sitter space introduced in [5] that has compactified region momentum space as boundary. When the region momenta are all space-like separated, the tetrahedron lies in a totally geodesic hyperbolic three-space (Euclidean $\mathrm{AdS}_{3}$ ) inside $\mathrm{AdS}_{5}$. It is a classical result that the volume of such an idea 2 tetrahedron is given by the Bloch-Wigner dilogarithm, and we show that this agrees with the evaluation of the four-mass box function in the physics literature $[1,24,25]^{3}$.

The scalar diagrams corresponding to box functions admit various degenerations where

\footnotetext{
${ }^{1}$ Twistors can in general be defined to be the chiral (perhaps pure) spinors of any conformal group.

${ }^{2} \mathrm{~A}$ tetrahedron in hyperbolic space is ideal if all four vertices lie at infinity.

${ }^{3}$ While the relation between box functions and volumes of geodesic tetrahedra in hyperbolic space has been noted before (see e.g. [25 28]), these authors took the hyperbolic space to be the mass-shell $p^{2}=m^{2}$ in momentum space. Geometrically, the hyperboloid $p^{2}=m^{2}$ lies entirely at infinity in the $\mathrm{AdS}_{5}$ considered in this paper, and its definition breaks dual conformal invariance.
} 
one or more corners of the box become massless. In these limits the box functions themselves diverge. This divergence can be seen clearly in the geometry, arising from the divergent volume of a tetrahedron when an entire edge goes to infinity (which occurs for each massless corner). The regularisation procedure of Alday \& Henn et al. [29,30] particularly naturally in this context as it is equivalent to regularising the tetrahedron's volume by bringing its vertices in from the infinity of $\mathrm{AdS}_{5}$ to lie on a finite surface, a horosphere. This procedure has a straightforward relationship with dimensional regularisation.

One loop amplitudes in $\mathcal{N}=4 \mathrm{SYM}$ can be expressed as a sum over various different boxes [22], with coefficients determined by their leading singularities [31]. The geometric point of view allows us to regard the sum of boxes as the 3-volume of the union of the constituent tetrahedra. In the simplest case of $n$-particle MHV amplitudes, we show that the corresponding tetrahedra fit together to form a 3-dimensional polytope without 2-boundary, embedded in $\mathrm{AdS}_{5}$. This gives a coherent geometrical realisation of the amplitude as the volume of a closed (piecewise linear) 3-manifold. Recent work [32] suggests that this correspondence between amplitudes and polytopes can be extended to higher MHV degree.

\section{Box functions as tetrahedra in $\mathrm{AdS}_{5}$}

The external momenta $p_{i}$ of an $n$-particle colour-ordered Yang-Mills amplitude may be encoded in $n$ region momenta $x_{i}$, defined up to overall translation by

$$
x_{i}-x_{i+1}=p_{i}, \quad x_{n+1} \equiv x_{1}
$$

so that momentum conservation is automatic. In terms of region momenta, the 4-mass box function is defined to bet

$$
F(i, j, k, l):=-\frac{N}{8 \pi^{2}} \int_{\mathbb{R}^{3,1}} \frac{\mathrm{d}^{4} x_{0}}{\left(x_{0 i}^{2}+\mathrm{i} \varepsilon\right)\left(x_{0 j}^{2}+\mathrm{i} \varepsilon\right)\left(x_{0 k}^{2}+\mathrm{i} \varepsilon\right)\left(x_{0 l}^{2}+\mathrm{i} \varepsilon\right)},
$$

where $N$ is a normalisation factor (determined below) and $x_{0}$ is the region momentum at the centre of the box (see figure 1). Following standard practice, we will implement the Feynman i $\varepsilon$-prescription by analytically continuing the integrand of (2) to the space of complexified region momenta, and rotating the contour of integration to the Euclidean real slice, where we may set $\varepsilon=0$.

It has long been known [1, 2] that when $\varepsilon=0$, the 4-mass box function is invariant under a 'dual' conformal symmetry that acts on the region momenta $x$ in the same way as the usual conformal transformations act on space-time coordinates. This dual conformal symmetry becomes far more significant in the $\mathcal{N}=4$ supersymmetric theory, as it is conjectured to extend (albeit anomalously) to the full planar amplitude [5, 6, 9] and may be viewed as the imprint of the integrability of the planar theory on scattering amplitudes [14,17].

\footnotetext{
${ }^{4}$ The factor $-1 / 8 \pi^{2}$ comes from the standard normalisation $-(4 \pi)^{2-\epsilon} /(2 \pi)^{4-2 \epsilon}$ of the dimensionally regularised box function (in the limit $\epsilon \rightarrow 0$ for the finite 4-mass box), together with the difference between our $N$ and the Gram determinant of e.g. 22.
} 


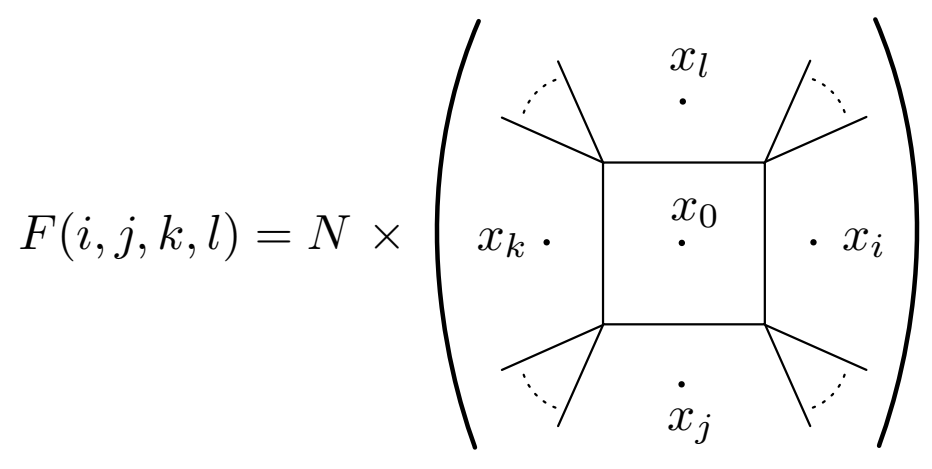

Figure 1: The 4-mass box function is defined by the integral (21). The region momenta are fixed up to overall translation by $x_{i}-x_{i+1}=p_{i}$, while the momentum running through a propagator is given by the difference of the region coordinates on either side of that propagator.

Dual conformal transformations do not act on the space $x$, because they can exchange a finite point with a point 'at infinity'. To make invariance manifest, we will re-express the region momenta in terms of skew matrices $X^{\alpha \beta}(\alpha, \beta=0, \ldots, 3)$, taking

$$
x^{A A^{\prime}} \rightarrow X^{\alpha \beta}=\left(\begin{array}{cc}
-\frac{1}{2} \epsilon^{A B} x^{2} & \mathrm{i} x_{B^{\prime}}^{A} \\
-\mathrm{i} x_{A^{\prime}}^{B} & \epsilon_{A^{\prime} B^{\prime}}
\end{array}\right) .
$$

Any matrix $X$ of this form satisfies

$$
X \cdot X:=\frac{1}{2} \epsilon_{\alpha \beta \gamma \delta} X^{\alpha \beta} X^{\gamma \delta}=0,
$$

while for two such matrices $X_{i}$ and $X_{j}$ we have

$$
X_{i} \cdot X_{j}=-\left(x_{i}-x_{j}\right)^{2}
$$

(We work with a $(+---)$ signature space-time, so that $X_{i} \cdot X_{j}>0$ if $i$ and $j$ are space-like separated.)

The special form of $X$ given in (3) really represents a particular coordinate patch of (dual) conformally compactified space-time. The full, compacitified space may be thought of as the quadric $X \cdot X=0$ in $\mathbb{R P}^{5}$, on which $X^{\alpha \beta} \sim \lambda X^{\alpha \beta}$ are homogeneous coordinates. This quadric has signatures $(2,4),(1,5)$ or $(3,3)$ (and hence topology $S^{1} \times S^{3}$, $S^{4}$ or $\left.\left(S^{2} \times S^{2}\right) / \mathbb{Z}_{2}\right)$ in Lorentzian, Euclidean or ultrahyperbolic signature space-times, respectively. Dual conformal transformations act on $\mathbb{R P}^{5}$ via the vector field

$$
J_{\beta}^{\alpha}=X^{\alpha \gamma} \frac{\partial}{\partial X^{\gamma \beta}},
$$

showing in particular that conformal transformations respect the linear structure of $\mathbb{R P}^{5}$ (whereas the linear structure of a Minkowski coordinate patch is not preserved).

The Feynman is prescription can be satisfied by analytically continuing the integrand to Euclidean signature and setting $\varepsilon=0$. We can therefore recast (2) as the contour 
integral

$$
F(i, j, k, l)=-\frac{N}{8 \pi^{2}} \oint_{S^{4}} \frac{D^{4} X}{X \cdot X_{i} X \cdot X_{j} X \cdot X_{k} X \cdot X_{l}},
$$

where the holomorphic 4-form $D^{4} X$ is defined as

$$
D^{4} X:=\frac{1}{\pi \mathrm{i}} \oint_{S^{1}} \frac{X \wedge \mathrm{d} X \wedge \mathrm{d} X \wedge \mathrm{d} X \wedge \mathrm{d} X \wedge \mathrm{d} X}{X^{2}}
$$

in terms of the canonical SL( $6, \mathbb{C})$-invariant top form of weight 6 on $\mathbb{C P}^{5}$. The $S^{1}$ contour in (8) is chosen to encircle the simple pole at $X^{2}=0$, and so restricts the remaining integral to the complex quadric $X \cdot X=0$. The $S^{4}$ contour in (17) is an integration over (dual) conformally compactified Euclidean space. Notice that we can replace the noncompact integral over Euclidean space (2) by an integral over $S^{4}$ because (2) is regular as $x_{0} \rightarrow \infty$, reflecting the UV finiteness of the original momentum space expression. The normalization factor $N$ is fixed by the requirement

$$
\frac{N}{(2 \pi \mathrm{i})^{4}} \oint_{\left(S^{1}\right)^{4}} \frac{D^{4} X}{X \cdot X_{i} X \cdot X_{j} X \cdot X_{k} X \cdot X_{l}}=1
$$

where the contour is now taken to compute the residue at each simple pole in the denominator (corresponding to cutting each of the four propagators in figure 11). This integral is straightforward 5 and yields

$$
N=\left|X_{i} \wedge X_{j} \wedge X_{k} \wedge X_{l}\right|
$$

where $|\cdot|$ denotes the norm induced on 4 -forms from the flat six-dimensional metric. Note that the sign of this normalisation factor depends on the orientation of the contour used to perform (9). This orientation is determined by an ordering of the points $X_{i}, X_{j}, X_{k}$ and $X_{l}$.

We can use the standard Feynman trick to reduce the denominator of (7) to a simple factor of degree 4. Introducing four real Feynman parameters $\alpha_{a} \in[0,1]=: I$, with $a=1, \ldots, 4$ one has

$$
F(i, j, k, l)=-\frac{6 N}{8 \pi^{2}} \int_{I^{4} \times S^{4}} \mathrm{~d}^{4} \alpha \delta\left(\sum_{a=1}^{4} \alpha_{a}-1\right) \frac{D^{4} X}{(X \cdot X(\alpha))^{4}},
$$

\footnotetext{
${ }^{5}$ The integral may be performed by first choosing two vectors $E, F \in \mathbb{C}^{6}$ that are null, perpendicular to the span of $X_{i}$, and obey $E \cdot F=1$. The affine coordinate chart of $\mathbb{C P}^{5}$ where $X \cdot F=1$ then has coordinates $y_{1}=X \cdot X_{i}, y_{2}=X \cdot X_{j}, \ldots, y_{5}=X \cdot E$. On this chart we can combine equations (8) and (9) to obtain the contour integral

$$
\begin{aligned}
\frac{(2 \pi \mathrm{i})^{4}}{N} & =\frac{1}{\pi \mathrm{i}} \frac{1}{\left|E \wedge F \wedge X_{i} \wedge X_{j} \wedge X_{k} \wedge X_{l}\right|} \oint \frac{\mathrm{d} y_{1} \wedge \mathrm{d} y_{2} \wedge \mathrm{d} y_{3} \wedge \mathrm{d} y_{4} \wedge \mathrm{d} y_{5}}{y_{1} y_{2} y_{3} y_{4}\left(2 y_{5} E \cdot F+Q(y, y)\right)} \\
& =\frac{1}{2 \pi \mathrm{i}} \frac{1}{\left|X_{i} \wedge X_{j} \wedge X_{k} \wedge X_{l}\right|} \oint \frac{\mathrm{d} y_{1}}{y_{1}} \wedge \frac{\mathrm{d} y_{2}}{y_{2}} \wedge \frac{\mathrm{d} y_{3}}{y_{3}} \wedge \frac{\mathrm{d} y_{4}}{y_{4}} \wedge \frac{\mathrm{d} y_{5}}{y_{5}}
\end{aligned}
$$
}

where $Q(y, y)$ is the part of the quadratic $X \cdot X$ involving only $y_{1}, \ldots, y_{4}$. This now leads directly to (10). 
where

$$
X(\alpha):=\alpha_{1} X_{i}+\alpha_{2} X_{j}+\alpha_{3} X_{k}+\alpha_{4} X_{l} .
$$

In this $\mathbb{R} \mathbb{P}^{5}$ framework, the individual propagators combine in a linear fashion, compared to the quadratic expression one finds in momentum space. The difference arises because in $\mathbb{R P}^{5}$, the propagator factors are linear in the homogeneous coordinates as a consequence of (5). In using the embedding in $\mathbb{R P}^{5}$, the tetrahedron of Feynman parameters, embedded via the $X(\alpha)$ of equation (12), lies in the interior of AdS rather than being restricted to lie on the boundary $X \cdot X=0$ as in earlier work, for example [25 28]. As we will see, the linearity of $X(\alpha)$ in the $\alpha_{a}$ implies that the embedding is totally geodesically for the AdS metric.

The $D^{4} X$ integral can be performed using momentum twistors. $X^{2}=0$ ensures that $X^{\alpha \beta}=A^{[\alpha} B^{\beta]}$ for some pair of linearly independent (momentum) twistors $A, B$. We can thus replace the $X$ integral by an integral over $A$ and $B$ separately

$$
\oint_{S^{4}} \frac{D^{4} X}{(X \cdot X(\alpha))^{4}}=\frac{1}{2 \pi \mathrm{i}} \oint_{\mathbb{C P}^{3}} \frac{D^{3} A D^{3} B}{\left(A^{\alpha} B^{\beta} X_{\alpha \beta}(\alpha)\right)^{4}}
$$

where the contour is over a copy of $\mathbb{C P}^{3} \subset \mathbb{C P}_{A}^{3} \times \mathbb{C P}_{B}^{3}$ defined by $A=\hat{B}$, where $\hat{B}$ denotes Euclidean complex conjugation

$$
Z^{\alpha}=\left(Z^{0}, Z^{1}, Z^{2}, Z^{3}\right) \mapsto \hat{Z}^{\alpha}=\left(-\overline{Z^{1}}, \overline{Z^{0}},-\overline{Z^{3}}, \overline{Z^{2}}\right) .
$$

Euclidean conjugation has no fixed points in twistor space, so on the contour $A=\hat{B}, A$ and $B$ never coincide and the integral on the right of (13) may be thought of as taken over the total space of the fibration

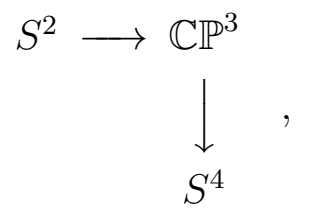

thus encoding the original integral over conformally compactified Euclidean space (the factor of $1 / 2 \pi \mathrm{i}$ in (13) compensates for the integration over the fibres; see [23] for further discussion). This integral can be evaluated as

$$
\oint_{\mathbb{C P}^{3}} \frac{D^{3} A D^{3} B}{\left(A^{\alpha} B^{\beta} X_{\alpha \beta}(\alpha)\right)^{4}}=\frac{1}{\operatorname{det} X(\alpha)} \oint_{\mathbb{C P}^{3}} \frac{D^{3} A D^{3} C}{\left(A^{\alpha} C_{\alpha}\right)^{4}}=\frac{(2 \pi \mathrm{i})^{3}}{6 \operatorname{det} X(\alpha)}
$$

where we have changed variables $B^{\beta} \rightarrow C_{\alpha}:=X_{\alpha \beta} B^{\beta}$ at the expense of a Jacobian, and then used the fact that the remaining integral is just $\mathrm{i}^{3}$ times the volume of $\mathbb{C P}^{3}$ in the Fubini-Study metric. Since $X(\alpha)$ is skew symmetric

$$
\operatorname{det} X(\alpha)=(\operatorname{Pfaff} X(\alpha))^{2}=\frac{1}{4}(X(\alpha) \cdot X(\alpha))^{2}
$$

where we recall that the dot product is defined by the four index $\varepsilon$-symbol that also defines the Pfaffian. Thus we obtain

$$
F(i, j, k, l)=2 \int_{I^{4}} \mathrm{~d}^{4} \alpha \delta\left(\sum_{a=1}^{4} \alpha_{a}-1\right) \frac{\left|X_{i} \wedge X_{j} \wedge X_{k} \wedge X_{l}\right|}{(X(\alpha) \cdot X(\alpha))^{2}} .
$$



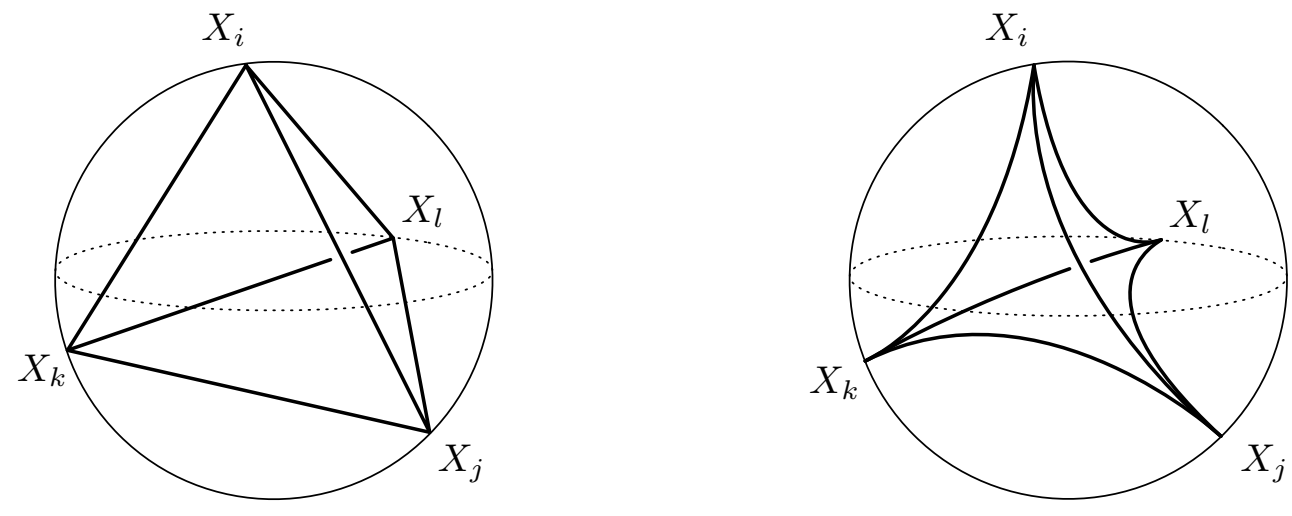

Figure 2: An ideal tetrahedron in AdS, shown in both the Klein-Beltrami (l) and Poincaré (r) models. All vertices lie on the conformal boundary at infinity, and each edge is an AdS geodesic.

for the Euclidean 4-mass box integral.

We now turn to a geometric interpretation of this formula. The expression

$$
X(\alpha)=\alpha_{1} X_{i}+\alpha_{2} X_{j}+\alpha_{3} X_{k}+\alpha_{4} X_{l}
$$

defines a linear map from the space of Feynman parameters, the unit simplex in $\mathbb{R}^{3}$, to $\mathbb{R} \mathbb{P}^{5}$. Provided the four vertices $X_{i}, X_{j}, X_{k}$ and $X_{l}$ are space-like separated (always true in Euclidean signature) we have $X(\alpha) \cdot X(\alpha)>0$, so we may take advantage of the projective invariance of (18) to introduce normalised coordinates

$$
Y(\alpha)=\frac{X(\alpha)}{\sqrt{X(\alpha) \cdot X(\alpha)}}
$$

obeying $Y \cdot Y=1$. Thus $Y$ defines a map from the space of Feynman parameters to Euclidean $\mathrm{AdS}_{5}$, i.e., the five dimensional hyperbolic ball. Straight lines in $\mathbb{R} \mathbb{P}^{5}$ are precisely the geodesics in $\mathrm{AdS}_{5}$, so as the $\alpha_{a}$ vary over the 3 -simplex $\left\{\alpha_{a} \in \mathbb{R}^{\geq 0} \mid \sum_{a=1}^{4} \alpha_{a}=1\right\}$, $Y(\alpha)$ varies over a tetrahedron in $\mathrm{AdS}_{5}$ whose vertices lies on the boundary at infinity, and whose edges and faces are totally geodesic. Such a tetrahedron is called ideal, and is depicted in figure 2,

We wish to show that $F(i, j, k, l)$ is simply (twice) the 3 -volume of this ideal tetrahedron. Using the delta function to eliminate $\alpha_{4}$, this volume can be written as the integral

$$
\operatorname{Vol}(i, j, k, l)=\int_{\text {tetra }} \mathrm{d}^{3} \alpha\left|\frac{\partial Y(\alpha)}{\partial \alpha_{1}} \wedge \frac{\partial Y(\alpha)}{\partial \alpha_{2}} \wedge \frac{\partial Y(\alpha)}{\partial \alpha_{3}}\right|
$$

where $|\cdot|$ denotes the norm on 3 -forms induced by the flat metric in $\mathbb{R}^{6} \supset \mathrm{AdS}_{5}$. Because $Y(\alpha)$ is normalised, each derivative $\partial Y(\alpha) / \partial \alpha^{a}$ is orthogonal to $Y(\alpha)$ itself, so

$$
\left|\frac{\partial Y(\alpha)}{\partial \alpha_{1}} \wedge \frac{\partial Y(\alpha)}{\partial \alpha_{2}} \wedge \frac{\partial Y(\alpha)}{\partial \alpha_{3}}\right|=\left|Y(\alpha) \wedge \frac{\partial Y(\alpha)}{\partial \alpha_{1}} \wedge \frac{\partial Y(\alpha)}{\partial \alpha_{2}} \wedge \frac{\partial Y(\alpha)}{\partial \alpha_{3}}\right|
$$


where now the norm is that on 4 -forms. The volume integral then reduces to

$$
\operatorname{Vol}(i, j, k, l)=\int_{\text {tetra }} \mathrm{d}^{3} \alpha \frac{\left|X_{i} \wedge X_{j} \wedge X_{k} \wedge X_{l}\right|}{(X(\alpha) \cdot X(\alpha))^{2}},
$$

which is exactly one half the expression for the 4-mass box function obtained above. Since four generic6 points span a 3-space, any given tetrahedron in fact lies inside some hyperbolic 3-space $\subset \mathrm{AdS}_{5}$. (When we consider amplitudes in section 4 it will be natural to add various box functions together but it is then important to remember that different tetrahedra lie in different three dimensional subspaces.)

Explicit formulæ for this volume have been known since Lobachevskii [33]. It may be succinctly expressed as

$$
\begin{aligned}
B(z) & :=\operatorname{Im}\left\{\operatorname{Li}_{2}(z)\right\}+\arg (1-z) \log |z| \\
& =\frac{1}{2}\left(\operatorname{Li}_{2}(z)-\operatorname{Li}_{2}(\bar{z})\right)+\frac{1}{4}(\log (1-z)-\log (1-\bar{z})) \log (z \bar{z}),
\end{aligned}
$$

where $B(z)$ is the 'Bloch-Wigner dilogarithm' (and $\operatorname{Li}_{2}(z)=-\int_{0}^{z} \log (1-t) \mathrm{d} t / t$ is the usual 'Spence' dilogarithm), whose argument $z$ is interpreted as the cross-ratio of the location of the four vertices on the boundary 2-sphere of the hyperbolic 3-space (see e.g. [34] for a review). The two-term dilogarithm identities show that $B(z)$ depends on the ordering of the four points in the cross-ratio only up to sign; equivalently, the sign of $B(z)$ depends on the orientation of the tetrahedron.

We can make contact with the physics literature expression for the 4-mass box [1,24] as follows. We note first that the span of $X_{i}, X_{j}, X_{k}, X_{l}$ in $\mathbb{R P}^{5}$ is an $\mathbb{R P}^{3}$ and that generically, $X \cdot X$ restricts to give a non-singular quadric (an $S^{2}$ in our Euclideanized setup) in this $\mathbb{R P}^{3}$. We can choose homogeneous coordinates $y_{i}, i=1, \ldots, 4$ on this $\mathbb{R P}^{3}$ so that

$$
\left.X \cdot X\right|_{\mathbb{R}^{3}}=y_{1} y_{2}-y_{3}^{2}-y_{4}^{2}
$$

In these coordinates, the natural parametrization of points on the quadric by a complex coordinate $\zeta$, defined up to a Möbius transformation, is given by

$$
\left(y_{1}, y_{2}, y_{3}, y_{4}\right)=(1, \zeta \bar{\zeta}, \operatorname{Re} \zeta, \operatorname{Im} \zeta)
$$

Since $X_{i}, X_{j}, X_{k}, X_{l}$ all lie on the quadric, they each correspond to a particular value of $\zeta$, and we can use the Möbius freedom to set

$$
\left(\zeta_{i}, \zeta_{j}, \zeta_{k}, \zeta_{l}\right)=(0,1, \infty, z)
$$

With these choices, we can now see that

$$
z \bar{z}=\frac{X_{i} \cdot X_{l} X_{j} \cdot X_{k}}{X_{i} \cdot X_{j} X_{l} \cdot X_{k}}, \quad \text { and } \quad(1-z)(1-\bar{z})=\frac{X_{i} \cdot X_{k} X_{l} \cdot X_{j}}{X_{i} \cdot X_{j} X_{k} \cdot X_{l}}
$$

The Lorentzian formula may be obtained by analytic continuation of the above Euclidean result, replacing $\bar{z}$ by $\tilde{z}$ and treating $z$ and $\tilde{z}$ as independent complex variables. To do so one must specifiy a branch of the dilogarithm, and the correct choice depends on the channel of the scattering process (see e.g. [25,35] for details).

\footnotetext{
${ }^{6}$ If the positions of the four vertices are not linearly independent, the normalization factor $\left|X_{i} \wedge X_{j} \wedge X_{k} \wedge X_{l}\right|$ vanishes and the volume is zero. This is obvious geometrically - the tetrahedron is squashed flat.
} 


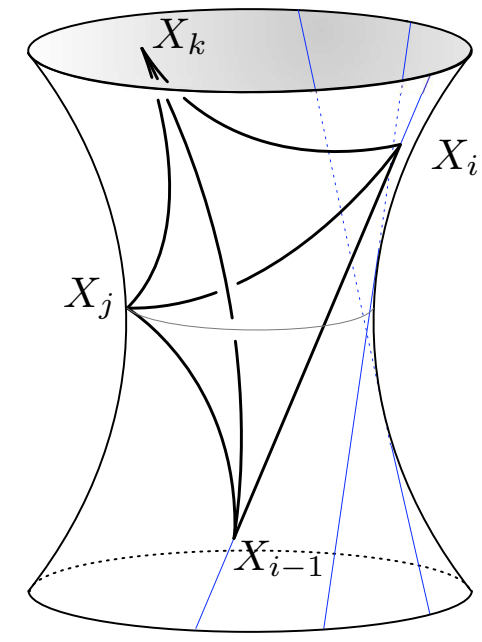

Figure 3: The 3-mass box integral has an entire edge along the boundary at infinity, so its volume diverges. This edge lies along a null geodesic (shown in blue) and the quadric $X \cdot X=0$ is ruled by these null lines.

\section{Regularisation of lower-mass box functions}

Infrared divergences in $\mathcal{N}=4$ super Yang-Mills amplitudes arise from degenerations of the 4-mass box function when there is just a single external massless particle attached to one or more corners of the box (in Lorentzian signature). Since the vertices each lie on the Klein quadric, the condition $0=p_{i-1}^{2}=\left(x_{i-1}-x_{i}\right)^{2}=X_{i} \cdot X_{i-1}=0$ implies that

$$
\left(X_{i-1}+\alpha X_{i}\right) \cdot\left(X_{i-1}+\alpha X_{i}\right)=0
$$

for any $\alpha$. Thus, an entire edge of the tetrahedra lies along infinity, causing the volume to diverge (see figure 3 ).

It is standard to regularise the divergence either by analytically continuing to $4-2 \epsilon$ dimensions, or by giving the external states a non-zero mass. In the present context, Henn et al. 229, 30, have shown that this latter mass regularisation may be understood as moving onto the Higgs branch of the theory. The resulting breaking of dual conformal invariance can be understood geometrically in terms of moving the points on the quadric $X \cdot X=0$ into the interior so that the geodesic tetrahedron that they determine clearly has finite volume. This can be done systematically by projecting from a chosen point at infinity onto a horosphere as follows.

Choose a point $I$ on the boundary of AdS that is space-like separated from each of the original vertices. $I$ will be the point at infinity in region momentum space and its choice breaks dual conformal invariance. There is a unique AdS geodesic through $I$ and each vertex and we can translate each vertex along the corresponding geodesic by replacing

$$
X_{i} \rightarrow X_{i}^{\prime}=X_{i}+\mu^{2}\left(I \cdot X_{i}\right) I
$$

where $\mu^{2}>0$ is a fixed parameter (see figure 44). The points $X_{i}^{\prime}, \ldots X_{l}^{\prime}$ then lie on the horosphere $X \cdot X=2 \mu^{2}(X \cdot I)^{2}$ in $\mathrm{AdS}_{5}$. 

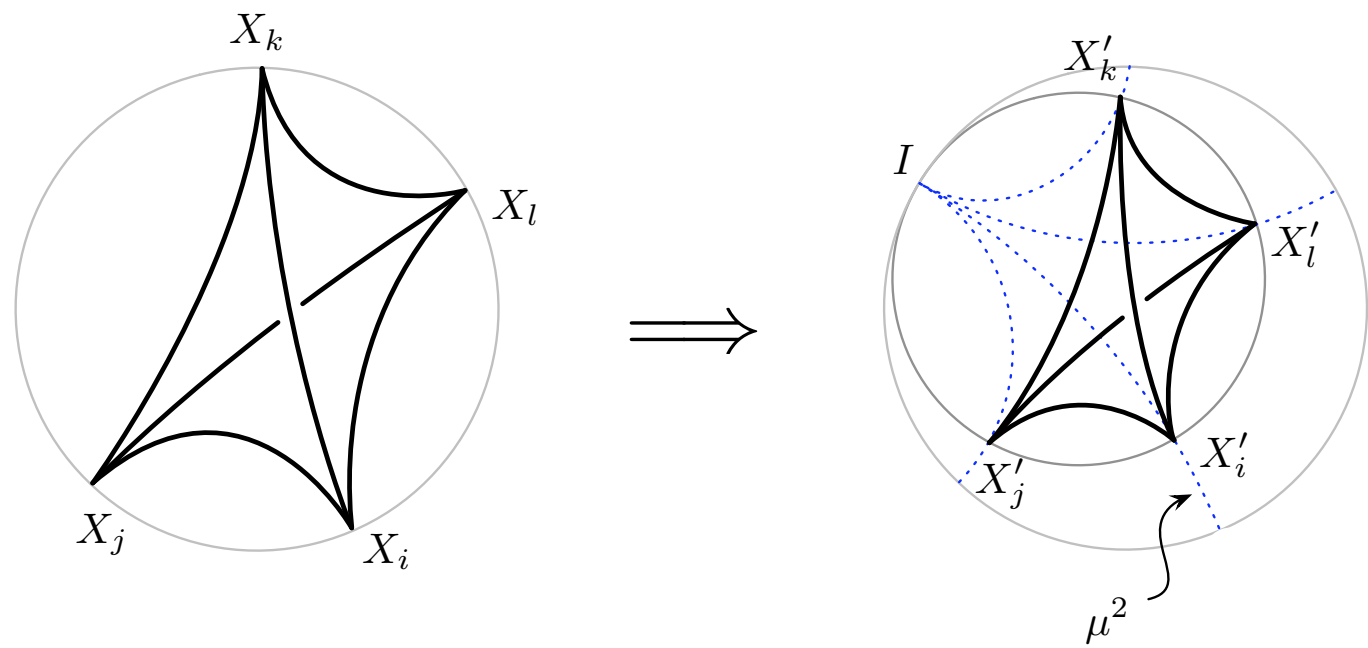

Figure 4: The tetrahedra may be regularised by bringing the vertices into the interior of AdS along the geodesic (shown in blue) connecting their original locations to a chosen point I. For simplicity, we can keep all the vertices on the same horosphere, corresponding to giving the external states equal masses.

With this replacement, the regularised box functions are defined by the same integrals as before, so that (e.g.) the regularised 3-mass box integral is 7

$$
F_{I, \mu^{2}}(i-1, i, j, k)=2 \int_{\bar{M}} D^{4} X \frac{\left|X_{i-1}^{\prime} \wedge X_{i}^{\prime} \wedge X_{j}^{\prime} \wedge X_{k}^{\prime}\right|}{X \cdot X_{i-1}^{\prime} X \cdot X_{i}^{\prime} X \cdot X_{j}^{\prime} X \cdot X_{k}^{\prime}} .
$$

All the manipulations that relate this integral to the tetrahedron 3-volume (23) (with vertices now at $X_{i}^{\prime}$ etc.) are unaffected. Since the tetrahedron no longer reaches the boundary, its volume is finite, but will depend on $I$ and $\mu^{2}$. Once again, explicit formulæ for the volume of an arbitrary (non-ideal) tetrahedron and, equivalently, for a scalar box function with arbitrary external masses are known, both in the mathematical [36, 37] and physical [25 27] literature, and have recently been re-derived by Hodges [23] from a point of view that is very close to the present paper. Here we just give those cases relevant to the one loop MHV amplitude. Keeping only terms that do not vanish as $\mu^{2} \rightarrow 0$, the regularised 2-mass easy box function (figure 5) may be written as

$$
\begin{aligned}
& F_{I, \mu^{2}}(i-1, i, j-1, j)= \\
& -\log \left(\frac{X_{i} \cdot X_{j}}{\mu^{2} X_{i} \cdot I X_{j} \cdot I}\right) \log \left(\frac{X_{i-1} \cdot X_{j-1}}{\mu^{2} X_{i-1} \cdot I X_{j-1} \cdot I}\right)+\frac{1}{2} \log ^{2}\left(\frac{X_{i} \cdot X_{j-1}}{\mu^{2} X_{i} \cdot I X_{j-1} \cdot I}\right) \\
& +\frac{1}{2} \log ^{2}\left(\frac{X_{j} \cdot X_{i-1}}{\mu^{2} X_{j} \cdot I X_{i-1} \cdot I}\right)+\operatorname{Li}_{2}\left(1-\frac{X_{i} \cdot X_{j-1} X_{i-1} \cdot I}{X_{i-1} \cdot X_{j-1} X_{i} \cdot I}\right)+\operatorname{Li}_{2}\left(1-\frac{X_{i} \cdot X_{j-1} X_{j} \cdot I}{X_{i} \cdot X_{j} X_{j-1} \cdot I}\right) \\
& +\operatorname{Li}_{2}\left(1-\frac{X_{i-1} \cdot X_{j} X_{j-1} \cdot I}{X_{i-1} \cdot X_{j-1} X_{j} \cdot I}\right)+\operatorname{Li}_{2}\left(1-\frac{X_{j} \cdot X_{i-1} X_{i} \cdot I}{X_{i} \cdot X_{j} X_{i-1} \cdot I}\right)-\operatorname{Li}_{2}\left(1-\frac{X_{i} \cdot X_{j-1} X_{j} \cdot X_{i-1}}{X_{i} \cdot X_{j} X_{i-1} \cdot X_{j-1}}\right)
\end{aligned}
$$

\footnotetext{
${ }^{7}$ The internal point $X$ still obeys $X \cdot X=0$.
} 

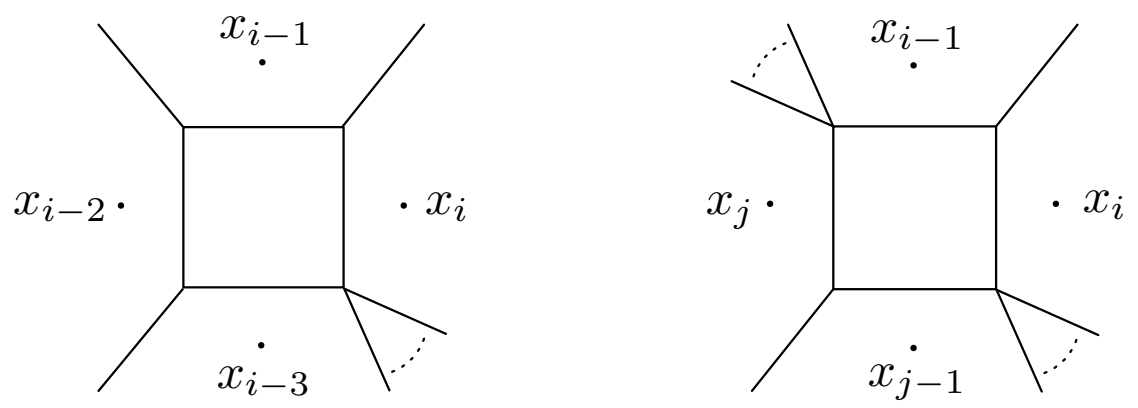

Figure 5: One loop MHV amplitudes involve only '1-mass' and '2-mass easy' box functions.

whereas the 1-mass box function is

$$
\begin{aligned}
& F_{I, \mu^{2}}(i-3, i-2, i-1, i)= \\
& -\log \left(\frac{X_{i-3} \cdot X_{i-1}}{\mu^{2} X_{i-3} \cdot I X_{i-1} \cdot I}\right) \log \left(\frac{X_{i-2} \cdot X_{i}}{\mu^{2} X_{i-2} \cdot I X_{i} \cdot I}\right)+\frac{1}{2} \log ^{2}\left(\frac{X_{i} \cdot X_{i-3}}{\mu^{2} X_{i} \cdot I X_{i-3} \cdot I}\right) \\
& +\operatorname{Li}_{2}\left(1-\frac{X_{i} \cdot X_{i-3} X_{i-1} \cdot I}{X_{i-1} \cdot X_{i-3} X_{i} \cdot I}\right)+\operatorname{Li}_{2}\left(1-\frac{X_{i} \cdot X_{i-3} X_{i-2} \cdot I}{X_{i-2} \cdot X_{i} X_{i-3} \cdot I}\right)+\frac{\pi^{2}}{6}
\end{aligned}
$$

and the zero-mass box function is

$$
F_{I, \mu^{2}}(1,2,3,4)=\log \left(\frac{X_{1} \cdot X_{3}}{\mu^{2} X_{1} \cdot I X_{3} \cdot I}\right) \log \left(\frac{X_{2} \cdot X_{4}}{\mu^{2} X_{2} \cdot I X_{4} \cdot I}\right)-\frac{\pi^{2}}{2} .
$$

We take the vertices of all our tetrahedra to lie on the same horosphere

$$
X \cdot X=2 \mu^{2}(X \cdot I)^{2}
$$

labelled by a single value of $\mu^{2}$. These formulæ may of course be simplified by normalising so that $X_{i} \cdot I=X_{j} \cdot I=\ldots=1$, but the expressions above serve to make the breaking of dual conformal invariance explicit. Terms in (32)-(34) that depend on $\mu^{2}$ are not invariant under a scale transformation of the boundary space-time, unless this is accompanied by a compensating rescaling of the regulator $\mu^{2}$. Terms that depend on cross-ratios involving the point $I$ are scale invariant, but not dual conformally invariant.

The mass regularisation discussed here is related to the more commonly used dimensional regularisation by a Mellin transform between the parameter $\mu$ and the $\varepsilon$ of dimensional regularization in dimension $4-2 \varepsilon$; see appendix B of [30] for details.

\section{One loop MHV amplitudes}

The one loop $n$-particle MHV amplitude in $\mathcal{N}=4$ SYM may be expressed [22] as a sum of 1-mass and 2-mass easy box functions (figure 5), each with coefficient one, times an overall factor of the $n$-particle MHV tree amplitude. Since each box function can be represented as a tetrahedron in $\mathrm{AdS}_{5}$, it is natural to ask how these tetrahedra fit together to give a geometric representation of the full one loop amplitude. 
Individual 2-mass easy or 1-mass box functions correspond to tetrahedra with vertices labelled by two consecutive pairs of integers

$$
(i-1, i, j-1, j) \quad \text { where } \quad\{i, j\} \in\{1, \ldots, n\}
$$

such that all four labels are distinct. There are thus $n 1$-mass boxes (where $j=i+2$ or $i=j+1$ working cyclically with $n+1=1)$ and $n(n-5) / 22$-mass easy boxes. The orientation of the tetrahedra determined by the ordering of their vertices in this list is thus unchanged under permutation of the pairs $(i-1, i) \leftrightarrow(j-1, j)$. This orientation also induces an orientation on each face of a tetrahedron, for a 2 -face is specified by a choice of three vertices, and we can declare the normal to the face to be 'inward' or 'outward' pointing depending on whether the omitted vertex lies in an odd or even position in the ordering given by (36).

To construct the $n$-particle 1-loop MHV amplitude, we must combine the volumes of these tetrahedra with coefficient one to obtain the volume of the union of all the tetrahedra. Perhaps surprisingly, the union is a 3-dimensional polytope without 2-boundary. The key point is that each 2-face is shared by precisely two tetrahedra, each inducing opposite orientations. In the generic case the 2 -face $(i, j-1, j)$ is shared by tetrahedra $(i-1, i, j-1, j)$ and $(i, i+1, j-1, j)$, each representing a 2 -mass easy box. In the first of these the omitted vertex $i-1$ is in an odd place in the labelling, whereas in the second, the omitted vertex $i+1$ is in an even position, so the orientations of the two tetrahedra are compatible. When $(i, j-1, j)$ are all consecutive (so $j-2=i$ ), the second tetrahedron is instead $(i, j-1, j, j+1)=(i, i+1, i+2, i+3)$, representing a 1 -mass box 8 . The gluing is again compatible with the orientation of the individual components.

The tetrahedra thus combine to form a closed 9 three dimensional polytope in $\mathrm{AdS}_{5}$, whose total 3-volume is proportional to the 1-loop MHV amplitude. For example, the five particle amplitude consists of the five possible 1-mass boxes and lies within an $\mathrm{AdS}_{4} \subset$ $\mathrm{AdS}_{5}$. The five corresponding tetrahedra join together to form the boundary of a 4simplex. For six particles, one finds a polyhedron with nine tetrahedral 3-faces that (for generic kinematics) cannot be restricted to live in any lower dimensional subspace of $\mathrm{AdS}_{5}$.

These amplitude polytopes have $n$ null edges that lie entirely on boundary of $\mathrm{AdS}_{5}$ (forming the polygonal Wilson loop) and hence their volume diverges. The regularisation discussed in section 3 is compatible with the picture of gluing the basic tetrahedra into a polyhedron provided we always use the same horosphere (i.e., we use the same reference point $I$ and parameter $\mu$ for each box). Thus the regularized amplitude will be a polytope with vertices brought in from infinity to lie on a horosphere. Performing the Mellin transform in the parameter $\mu$ as in appendix B of [30] will then give the full dimensionally regularized amplitude.

\footnotetext{
${ }^{8}$ Note that tetrahedron $(i, j-1, j, j+1)$ is not available when $j \neq i+2$ as it then represents a 2 -mass hard box function, which does not contribute to the MHV amplitude.

${ }^{9}$ The four particle one-loop MHV amplitude, involving a single, zero mass box function, seems to be exceptional in this regard, although even this can be thought of as two copies of the same tetrahedron and so is still closed, albeit degenerate.
} 


\section{Discussion}

General, one-loop $\mathrm{N}^{k} \mathrm{MHV}$ amplitudes in $\mathcal{N}=4 \mathrm{SYM}$ are always expressible as linear combinations of box functions, so a 3-volume interpretation is still possible. However, beyond MHV the situation is complicated by the fact that different box functions have different leading singularities as coefficients [31]. If one factors the 1-loop MHV amplitude out of the complete superamplitude, the ratio is conjectured to be dual conformally invariant and finite [9]. Intriguing evidence has emerged [32] that each finite combination of boxes that then appears with a particular coefficient corresponds to the volume of a 3 -polytope in $\mathrm{AdS}_{5}$. Furthermore, just as for the MHV amplitude itself, these polytopes are believed to have no 2-boundary.

Following the tree-level discussion in [18], it should be possible to understand the cancellation of spurious singularities at one loop also. The situation is more complicated here and there are different types of singularities that arise, and that can be spurious. For example, the most dominant singularities of a box function are are the infrared sungularities. These are associated to edges which go to infinity when the pairs of vertices at each end become null separated. According to the standard infrared properties of one loop amplitudes we have in dimensional regularization

$$
\left.M_{n}^{1-\mathrm{loop}}\right|_{\mathrm{IR}}=-\frac{1}{\epsilon^{2}} M_{n}^{\text {tree }} \sum_{i=1}^{n}\left(X_{i} \cdot X_{i+2}\right)^{\epsilon}
$$

and so the physical singularities correspond precisely to those edges connecting vertex $i$ to vertex $i+2$, and those corresponding to the other edges must therefore be spurious.

We expect that $\ell$-loop amplitudes can similarly be associated with volumes of higher dimensional polytopes embedded in the $\ell$-fold Cartesian product of $\mathrm{AdS}_{5}$. The basic geometry associated to the use of Feynman parameters leading to polydtopes in copies of $\mathbb{R} \mathbb{P}^{5}$ would seem to extend strightforwardly to higher loops. Again, the scheme here is reminiscent of the description of NMHV tree amplitudes as volumes of polytopes in momentum twistor space [18]. Although it is clear that it should be possible to describe $\mathrm{N}^{k} \mathrm{MHV}$ amplitudes as volumes in the $k$-fold product of momentum twistor space, it is simpler to use instead a dual description in terms of residues in Grassmannians [19,20]. It therefore seems plausible that the $\mathbb{R P}^{5}$ description of one loop boxes should extend most naturally to a Grassmannian description of higher loop integrals.

\section{Acknowledgements}

It is a pleasure to thank Nima Arkani-Hamed, Freddy Cachazo, James Drummond, Johannes Henn and Andrew Hodges for many useful discussions, and the Faculty and Staff of IAS, Princeton for hospitality while this work was carried out. The work of DS is supported by the Perimeter Institute for Theoretical Physics. Research at the Perimeter Institute is supported by the Government of Canada through Industry Canada and by the Province of Ontario through the Ministry of Research \& Innovation. The work of LM was financed in part by EPSRC grant number EP/F016654, see also http://gow . epsrc .ac.uk/ViewGrant . aspx?GrantRef=EP/F016654/1. 


\section{References}

[1] A. Davydychev and N. Usyukina, An approach to the evaluation of three and four point ladder diagrams, Phys. Lett. B298 (1993) 363-370.

[2] D. Broadhurst, Summation of an infinite series of ladder diagrams, Phys. Lett. B307 (1993) 132-139.

[3] J. Drummond, J. Henn, V. Smirnov, and E. Sokatchev, Magic identities for conformal four-point integrals, JHEP 01 (2007) 064, hep-th/0607160.

[4] Z. Bern, J. J. Carrasco, H. Johansson, and D. Kosower, Maximally Supersymmetric Planar Yang-Mills Amplitudes at Five Loops, Phys. Rev. D76 (2007) 125020, [arXiv:0705.1864.

[5] L. F. Alday and J. Maldacena, Gluon scattering amplitudes at strong coupling, JHEP 06 (2007) 064, arxiv 0705.0303 [hep-th]].

[6] J. Drummond, J. Henn, G. Korchemsky, and E. Sokatchev, Conformal Ward identities for Wilson loops and a test of the duality with gluon amplitudes, Nucl. Phys. B826 (2010) 337-364, arXiv:0812.1223 [hep-th]].

[7] A. Brandhuber, P. Heslop, and G. Travaglini, A Note on Dual Superconformal Symmetry of the $\mathcal{N}=4$ Super-Yang-Mills S-Matrix, Phys. Rev. D78 (2008) 125005, [arXiv:0807.4097].

[8] J. M. Drummond and J. M. Henn, All Tree-Level Amplitudes in $\mathcal{N}=4$ SYM, JHEP 04 (2009) 018, arXiv:0808.2475].

[9] J. M. Drummond, J. Henn, G. P. Korchemsky, and E. Sokatchev, Dual Superconformal Symmetry of Scattering Amplitudes in $\mathcal{N}=4$ Super-Yang-Mills Theory, arXiv:0807.1095.

[10] H. Elvang, D. Z. Freedman, and M. Kiermaier, Dual conformal symmetry of 1-loop NMHV amplitudes in $\mathcal{N}=4$ SYM theory, JHEP 03 (2009) 075, arXiv:0905.4379 [hep-th].

[11] A. Brandhuber, P. Heslop, and G. Travaglini, Proof of the dual conformal anomaly of one-loop amplitudes in $\mathcal{N}=4$ SYM, JHEP 10 (2009) 063, arXiv:0906.3552 [hep-th].

[12] N. Beisert, R. Ricci, A. Tseytlin, and M. Wolf, Dual superconformal symmetry from $A d S_{5} \times S^{5}$ superstring integrability, Phys. Rev. D78 (2008) 126004, arXiv:0807.3228 [hep-th].

[13] N. Berkovits and J. Maldacena, Fermionic T-duality, dual superconformal symmetry and the amplitude/Wilson loop connection, JHEP 09 (2008) 062, arXiv:0807.3196 [hep-th]. 
[14] J. Drummond, J. Henn, and J. Plefka, Yangian symmetry of scattering amplitudes in $\mathcal{N}=4$ super Yang-Mills, JHEP 05 (2009) 046, arXiv:0902.2987 [hep-th].

[15] L. F. Alday and J. Maldacena, Minimal surfaces in AdS and the eight gluon scattering amplitude at strong coupling, arXiv:0903.4707 [hep-th].

[16] L. F. Alday, D. Gaiotto, and J. Maldacena, Thermodynamic Bubble Ansatz, arXiv:0911.4709 [hep-th].

[17] L. F. Alday, J. Maldacena, A. Sever, and P. Vieira, Y-system for Scattering Amplitudes, arXiv:1002.2459 [hep-th].

[18] A. Hodges, Eliminating Spurious Poles from Gauge-Theoretic Amplitudes, arXiv:0905.1473 [hep-th].

[19] L. Mason and D. Skinner, Dual Superconformal Invariance, Momentum Twistors and Grassmannians, JHEP 11 (2009) 045, [arXiv:0909.0250].

[20] N. Arkani-Hamed, F. Cachazo, C. Cheung, and J. Kaplan, A Duality For The S Matrix, arXiv:0907.5418.

[21] N. Arkani-Hamed, F. Cachazo, and C. Cheung, The Grassmannian Origin Of Dual Superconformal Invariance, arXiv:0909.0483.

[22] Z. Bern, L. Dixon, D. Dunbar, and D. Kosower, One-loop n-point gauge theory amplitudes, unitarity and collinear limits, Nucl. Phys. B425 (1994) 217-260, hep-ph/9403226.

[23] A. Hodges, The box integrals in momentum-twistor geometry, To appear.

[24] A. Isaev, Multi-loop Feynman integrals and conformal quantum mechanics, Nucl. Phys. B662 (2003) 461-475, hep-th/0303056].

[25] A. Denner, U. Nierste, and R. Scharf, A compact expression for the scalar one loop four point function, Nucl. Phys. B367 (1991) 637-656.

[26] G. 't Hooft and M. Veltman, Scalar one-loop integrals, Nucl. Phys. B153 (1979) $365-401$.

[27] A. Davydychev and R. Delbourgo, A geometrical angle on Feynman integrals, J. Math. Phys. 39 (1998) 4299-4334, hep-th/9709216.

[28] A. Gorsky and A. Zhiboedov, One-loop derivation of the Wilson polygon - MHV amplitude duality, J. Phys. A42 (2009) 355214, arXiv:0904.0381.

[29] L. F. Alday, J. Henn, J. Plefka, and T. Schuster, Scattering into the fifth dimension of $\mathcal{N}=4$ super Yang-Mills, JHEP 01 (2010) 077, arXiv:0908.0684 hep-th.

[30] J. Henn, S. Naculich, H. Schnitzer, and M. Spradlin, Higgs-regularized three-loop four-gluon amplitude in $\mathcal{N}=4 S Y M$ : exponentiation and Regge limits, arXiv:1001.1358. 
[31] R. Britto, F. Cachazo, and B. Feng, Generalized unitarity and one loop amplitudes in $\mathcal{N}=4$ super Yang-Mills, Nucl. Phys. B725 (2005) 275-305, hep-th/0412103.

[32] N. Arkani-Hamed, J. Bourjaily, F. Cachazo, and J. Trnka, Private communication.

[33] J. Milnor, Hyperbolic geometry: the first 150 years, Bull. Amer. Math. Soc. 6 (1982) 9-24.

[34] D. Zagier, The dilogarithm function, in Frontiers in number theory, physics and geometry, vol. II, pp. 3-65, Springer, 2007.

[35] G. Duplancic and B. Nizic, Ir finite one-loop box scalar integral with massless internal lines, Eur. Phys. J. C24 (2002) 385-391, hep-ph/0201306].

[36] Y. Cho and H. Kim, On the volume formula for hyperbolic tetrahedra, Discrete $\mathcal{E}$ Comput. Geom. 22 (1999) 347-366.

[37] J. Murakami and M. Yano, On the volume of a hyperbolic and spherical tetrahedron, Commun. Anal. Geom. 13 (2005) 379-400. 